\title{
Estudio teórico de endocanabinoides análogos a anandamida ${ }^{1}$
}

Theoretical study analog a endocannabinoids anandamide

Estudo teórico análogo a anandamida endocanabinóides

Recibido: octubre de 2012

Aprobado: diciembre de 2012
Luis Carlos García Sánchez ${ }^{2}$

Julie Gesselle Benavides Melo ${ }^{3}$

Fabio Mayorga ${ }^{4}$

\section{Resumen}

A partir de métodos computacionales, se estudió un total de 90 reacciones diseñadas por el grupo de investigación de farmoquímica de la UPTC cuya finalidad es la síntesis y determinación de posible actividad farmacológica de derivados de la anandamida en la enfermedad de Parkinson. Se proponen dos tipos de reacción. En el primero se parte del ácido araquidónico y se pretende sustituir el grupo hidroxilo por diferentes especies (14 en total). En el segundo se parte de una estructura de ariletanolamina en la que se realizan sustituciones de posición y grupo funcional (16entotal). Cada una de las reacciones se realiza bajo la influencia de diferentes medios como cloruro de tionilo -SOCl2-, NN-diciclohexilcarboiimida -DCC- y acetonitrilo -CH3CN-. Para determinar cuál de los caminos de reacción propuestos brinda las mejores condiciones en cuanto a favorabilidad, tiempo y rendimiento, se realizaron cálculos AB-Initio y DFT (Density Functional Theory) empleando la base 3-21G. Los resultados obtenidos permiten afirmar que 16 de las reacciones estudiadas son las más viables y que los cálculos realizados por metodología DFT son los que presentan mayor correlación entre lo teórico y lo experimental.

Palabras clave: química teórica, endocannabinoides, anandamida.

\section{Abstract}

From computational methods, we studied a total of 90 reactions designed by the research group of AAUCTU pharmochemical aimed at determining the possible synthesis and pharmacological activity of anandamide derivatives in Parkinson's disease. We propose two types of reaction. In the first, part of arachidonic acid and is intended to replace the hydroxyl group by different species (14 in total). In the second part of an arylethanolamine structure in which substitutions are made position and functional group (16 in total). Each of the reactions is carried out under the influence of different media such as thionyl chloride, SOCl2-, NN-Diciclohexilcarboiimida-DCC-and-CH3CN-Acetonitrile. To determine which of the proposed reaction pathways, provides the best conditions in terms of favorability, time and performance calculations were

1 Artículo de Investigación financiado por el Centro de Investigaciones y Desarrollo Científico de la Universidad Distrital Francisco José de Caldas.

2 Profesor del Proyecto Curricular de Licenciatura en Química, coordinador de Laboratorio, Universidad Distrital Francisco José de Caldas. Bogotá - Colombia. Contacto: lcgarcia@udistrital.edu.co

3 Pontificia Universidad Católica de Chile. Santiago de Chile - Chile. Contacto: juliebenavidesm@gmail.com

4 Universidad Pedagógica y Tecnológica de Colombia, Tunja - Colombia. Contacto: fabio.mayorga@uptc.edu.co 
made AB-Initio and DFT (Density functional theory), using the 3-21G basis. The results confirm that 16 of the studied reactions are the most viable and calculations by DFT methodology are those with higher correlation between the theoretical and the experimental.

Keywords: theoretical chemistry, endocannabinoids, anandamide.

\section{Resumo}

A partir de métodos computacionais, estudamos um total de 90 reações projetados pelo grupo de pesquisa de AAUCTU farmoquímica objetivo determinar a possível síntese e atividade farmacológica de derivados anandamida na doença de Parkinson. Propomos dois tipos de reação. Na primeira, parte do ácido araquidônico e destina-se a substituir o grupo hidroxilo por diferentes espécies ( 14 no total). Na segunda parte de uma estrutura de ariletanolamina, em que as substituições são feitas posição e grupo funcional (16 no total). Cada uma das reacções é levada a cabo sob a influência de diferentes meios, tais como cloreto de tionilo, SOCI2-, NN-Diciclohexilcarboiimida-DCC-e-CH 3 CN-acetonitrilo. Para determinar quais das vias de reacção propostas, proporciona as melhores condições em termos de favorecimento, cálculos de tempo e de desempenho foram realizadas Ab initio e DFT (teoria funcional da densidade), utilizando a base de 3-21G. Os resultados confirmam que 16 das reações estudadas são a mais viável e cálculos da metodologia DFT são aqueles com maior correlação entre o teórico eo experimental.

Palavras-chave: química teórica, endocanabinóides, anandamida.

\section{Introducción}

La planta Cannabis sativa ha sido cultivada debido a sus presuntas propiedades medicinales y psicoactivas. El $\Delta 9$-THC (tetrahidrocannabinol) es conocido como su constituyente principal y en los humanos este sería el responsable de inducir cambios en el comportamiento, la percepción del tiempo, sonido, color y sabor; además provoca efectos en sitios con actividad motora y control de la postura que probablemente residen en el ganglio basal y el cerebelo, áreas que se encuentran densamente pobladas con sitios ligados a cannabinoides. La marihuana en el campo médico ha sido utilizada debido sus propiedades como relajante muscular, analgésico, antiinflamatorio, antiespasmódico, ansiolítico, broncodilatador, reductor de presión intraocular, anticonvulsivante e incluso ha sido utilizada por pacientes con cáncer como antiemético después de sus tratamientos de quimioterapia.

En la actualidad sigue siendo empleada en el tratamiento de diversas enfermedades, tales como glaucoma, asma, cáncer, migraña, insomnio, náuseas, esclerosis múltiple, molestias ocasionadas por neuropatías periféricas, entre otros padecimientos neuromusculares. $\mathrm{Y}$ se ha demostrado que su acción benéfica tiene origen en la presencia de $\Delta 9$-THC, razón por la cual se ha dado origen a ciertos derivados de carácter sintético.

Por las razones mencionadas anteriormente se incrementó el interés de la comunidad científica por el estudio de esta especie, sus principales constituyentes, sus características y su mecanismo de acción. Esto permitió el descubrimiento de la anandamida. Inicialmente, el químico israelí Raphael Mechoulam consiguió aislar el principio activo de la planta Cannabis sativa, es decir, eltetrahidrocannabinolo $\Delta 9$-THC; además, logró explicar sus mecanismos de acción. En 1988 fueron descubiertos los receptores específicos para el THC por William Devane y colaboradores y en 1990 Lisa Matsuda y sus colaboradores clonaron estos receptores para ser estudiados. ElTHC no fue encontrado naturalmente en humanos pero 
la resistencia de un receptor específico para el mismo hizo pensar que este era una imitación de un ligando natural molecular.

Mechoulam, en 1992, logró identificar una sustancia en el cerebro que actuó sobre el receptor de los cannabinoides. Se trataba de un ácido graso polinsaturado con un enlace químico del tipo amida al que denominó anandamida. Esta es una sustancia producida y empleada por el cerebro que cuenta con un perfil farmacológico similar al que induce $\Delta$ 9-THC, es decir, produce alteraciones en la consolidación de la memoria, hipomotilidad, nocicepción, hipotermia, entre otras. Razón por la cual se le denominó endocannabinoide.

La anandamida es químicamente conocida como araquidonil etanolamida. Se trata de un compuesto endógeno humano derivado del ácido araquidónico, que cuenta con actividad cannabinoide. Su acción es mediada por receptores cannabinoides CB1 (localizados principalmente en el sistema nervioso central) y CB2 (en el periférico) que se encuentran acoplados a proteínas G. Es un compuesto lábil, liposoluble, estable a $-20^{\circ}$ Cen solución con etanol; fue aislado y purificado por técnicas cromatográficas y espectroscópicas. Se ha demostrado la importancia que tiene dicho compuesto en el desarrollo humano, porque participa en el control del movimiento, memoria, emociones, regulación del sueño, temperatura corporal, modulación del dolor, respuesta de coagulación y respuesta inmune. Se conoce también que la anandamida produce los mismos efectos que los cannabinoides de origen vegetal: activa funciones cerebrales ligadas a la percepción, el aprendizaje, la memoria, el estado de ánimo, el movimiento, la secreción de hormonas, etc. Se piensa que la anandamida interviene en la formación de la memoria a corto plazo, ya que en la región cerebral del hipocampo hay una gran proporción de receptores cannabinoides. Pero lo que ha despertado nuestro interés principal es que se ha descubierto que puede inhibir la liberación de dopamina estriatal, lo que ha conducido a pensar que el bloqueo de los receptores cannabinoide CB1 permitiría proteger la adecuada liberación de dopamina estriatal, o al menos dificultará la gran caída de dicho neurotransmisor a los bajos niveles típicos en la enfermedad de Parkinson y ello podría colaborar en el tratamiento paliativo de dicha patología.

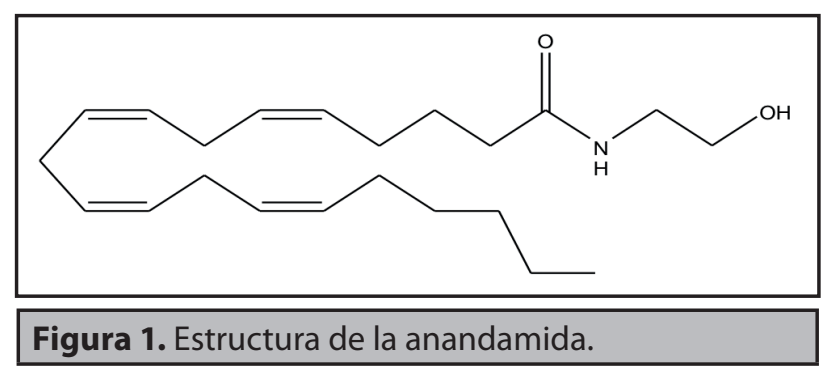

Fuente: elaboración propia.

Con base en los antecedentes mencionados sobre la influencia que tiene "la marihuana endógena" en la regulación y liberación de dopamina, además de las investigaciones desarrolladas por Piomelli y colaboradores (Neurosciences Institute of San Diego) en las que se concluye que la degradación de la anandamida puede ser inhibida por componentes del tipo $\mathrm{N}$-aciletanolaminas contenidos en el chocolate. Se diseñaron 90 reacciones para ser estudiadas teóricamente con el objetivo de depurar este número y considerar cuáles de ellas serían las más viables. Posteriormente serán sintetizadas y ensayadas farmacológicamente aquellas que hayan salido favorecidas por el estudio teórico. Dentro de las reacciones propuestas se encuentran derivados de la anandamida (también una $\mathrm{N}$-aciletanolamina) con alguna característica que les confiere la posibilidad de ejercer como antagonistas competitivos de los receptores CB1.

El estudio teórico fue desarrollado mediante la implementación del paquete computacional Gaussian comparando cálculos AB-Initio vs. DFT (Density Functional Theory) con una base 3-21 G.

\section{Sección experimental}

Las reacciones propuestas involucran compuestos que están organizados en dos grupos principales. El grupo A contiene derivados de ácido araquidónico (ver reacción general I) y el grupo B derivados de ariletanolaminas (ver reacción general II). Se optimiza la totalidad de moléculas propuestas, con el fin de obtener la estructura de mínima energía. 
Estructura que fue confirmada al observar las frecuencias de las mismas, por lo que se puede afirmar que en ninguno de los casos se trabajó con un estado de transición. Para todos los cálculos se utilizó la base 3-21G tanto en metodología AB-Initio como en DFT y en esta última se trabajó bajo el método de cálculo B3LYP.

Fue optimizada la totalidad de moléculas propuestas, con el fin de obtener la estructura de mínima

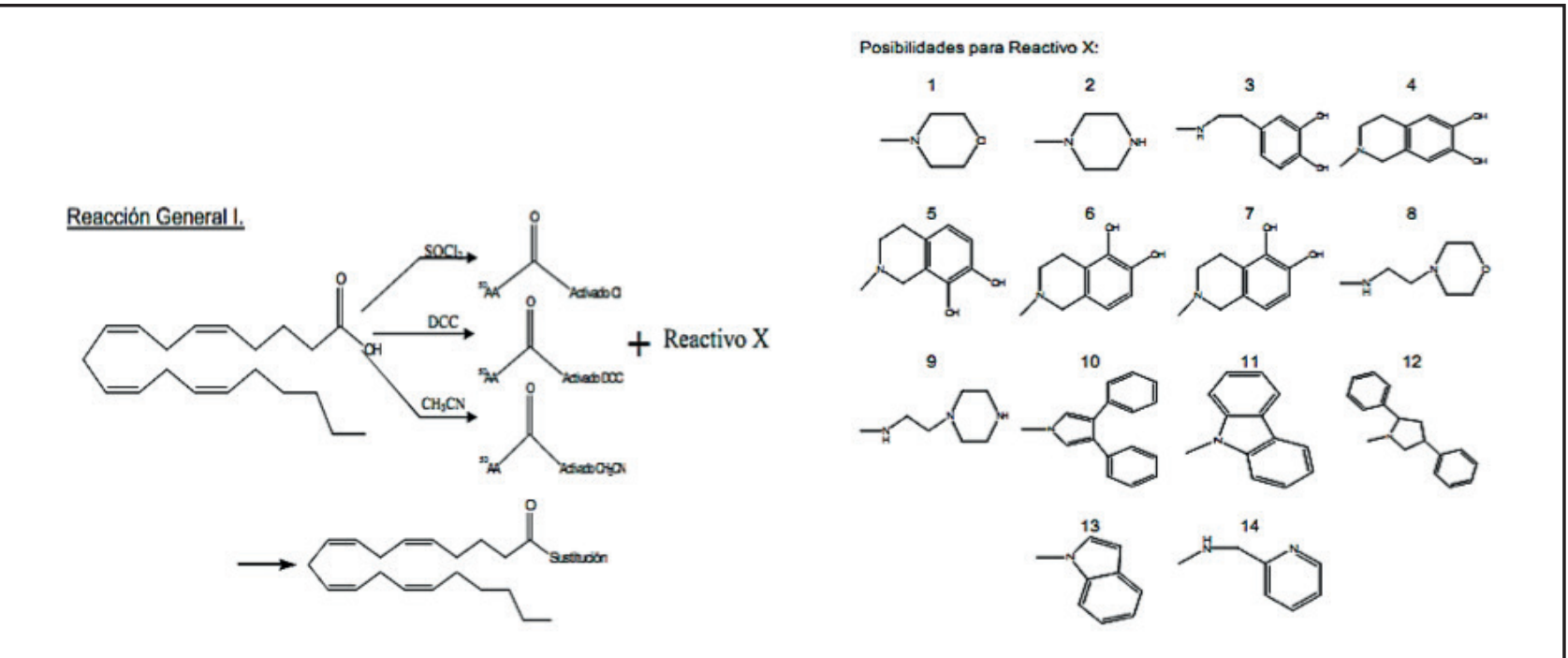

Figura 2. Derivados de ácido araquidónico.

Fuente: elaboración propia.
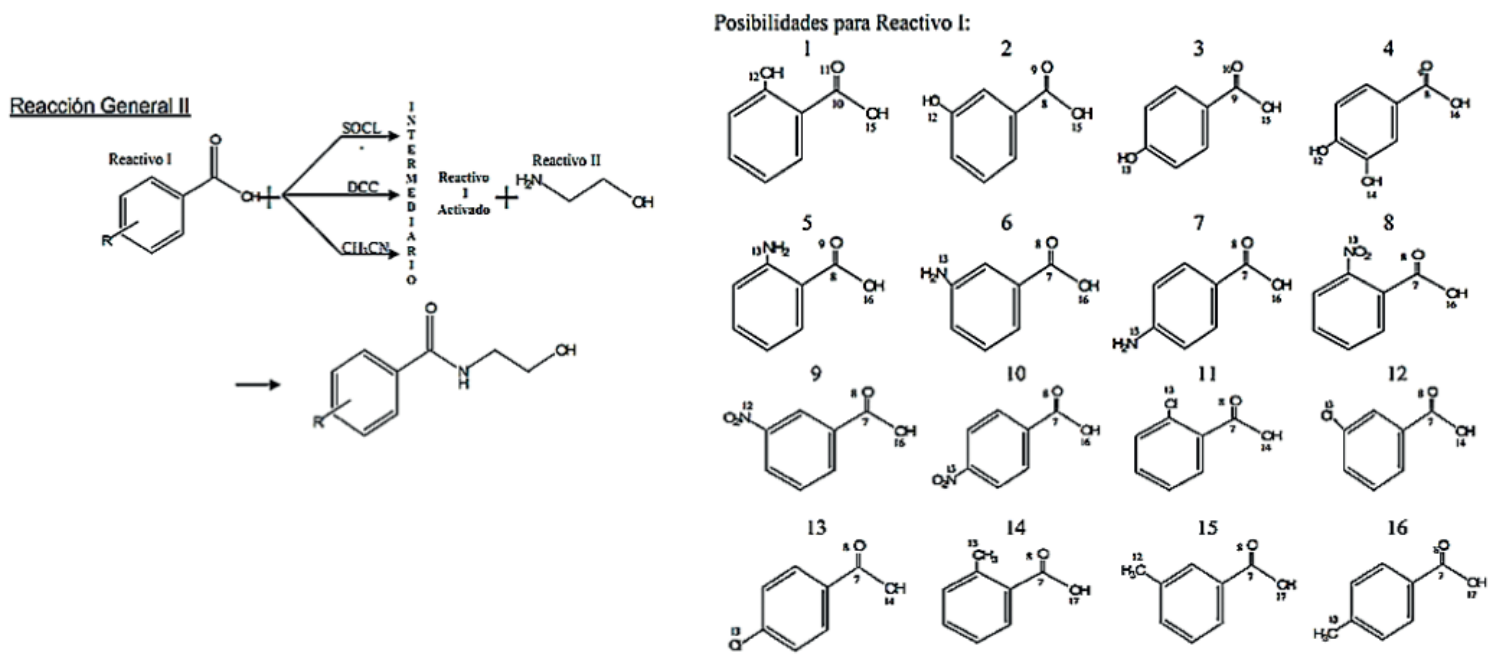

Figura 3. Derivados de ariletanolaminas

Fuente: elaboración propia. 
energía. Las estructuras fueron confirmadas al observar frecuencias positivas de las mismas, por lo que se puede afirmar que en ninguno de los casos se trabajó con un estado de transición. Para todos los cálculos se utilizó la base 3-21G tanto en metodología AB-Initio como en DFT y en esta última se trabajó bajo el método de cálculo B3LYP. Los parámetros de reactividad fueron realizados por análisis poblacional, comparando cargas de Mulliken y energías de orbitales de frontera propios para cada reacción.

\section{Resultados y discusión}

Inicialmente se parametriza el trabajo empleando las reacciones del artículo "Head Group Analogs of Arachidonylethanolamide, the Endogenous Cannabinoid Ligand", debido a que los reactivos involucrados en este son muy similares en cuanto a su estructura y comportamiento con relación a los compuestos propuestos. En esta parte de la investigación se procede a tomar las doce reacciones provenientes del artículo mencionado (ver uno de los ejemplos en la figura 3) y se dibuja cada una de las especies en diferentes paquetes computacionales (Hyperchem, WinMopac y ChemSketch). Se realizan cálculos bajo diversas metodologías (semiempíricos, Ab-Initio, híbridas) y, finalmente, se comparan los resultados obtenidos con los datos experimentales, evidenciando que los cálculos Ab-Initio y DFT son los más consistentes puesto que se correlacionan mucho mejor con los resultados experimentales y cuentan con mayor regularidad que los cálculos semiempíricos, aunque estos últimos tengan un menor costo computacional.Después de la parametrización se procede con el análisis de las reacciones propuestas. Y para el análisis poblacional se propone el siguiente procedimiento:

\section{Elaborar un posible mecanismo de reacción.}

2. Proceder con la elección de los átomos que se consideren como centros de reactividad, basándose en la propuesta de mecanismo ya elaborada.

3. Establecer el orbital del cual se extraerá la información (HOMO cuando se trata del átomo que está dispuesto a ceder electrones y LUMO cuando sea el que va a recibirlos).

4. Por último, tomar los valores elegidos y compararlos entre sí para llegar a predecir la ocurrencia de reacción.

La metodología propuesta fue empleada en cada una de las reacciones. Los datos recopilados confirman la posibilidad de lograr que la mayor parte de las reacciones transcurra con éxito, debido a que los valores reportados muestran congruencia entre los orbitales dispuestos a ceder carga y los
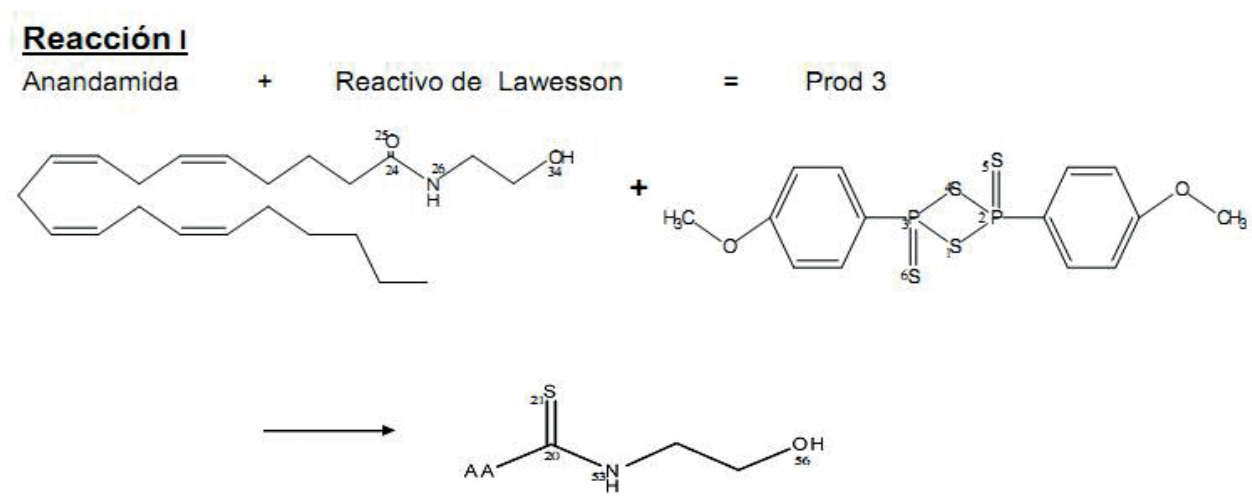

Figura 4. Ejemplo de reacciones análogas utilizadas en la parametrización.

Fuente: elaboración propia. 
encargados de recibirla (ver ejemplo en figura 6). Las reacciones $2,7,11,12,13$ y 14 para derivados de ácido araquidónico presentan magnitudes altas encada uno de los orbitales evaluados; mientras que en las reacciones con ariletanolaminas este mismo comportamiento sucede en las reacciones $1,2,3,4,6,7,11,12,13,14,15$ y 16 , por lo que se recomienda que estas sean privilegiadas durante la fase experimental de la investigación, pues serían las que transcurran con mayor eficiencia y alto rendimiento.

Los cálculos para las reacciones 3,5 y 9 de derivados de ácido araquidónico y 8, 9 y 10 en ariletanolaminas arrojaron valores bastante pequeños para los átomos de interés, así como magnitudes elevadas en otros lugares de la molécula. Razón por la cual se puede pensar que sería difícil que la reacción siga el curso esperado. Es probable que el ataque no surta efecto o que lo haga con influencia de otro átomo que se comporte como centro de reacción. Aunque se observa que la mayoría de posibilidades son efectivas, parece ser que el camino de reacción se ve favorecido cuando se utiliza SOCl2 y DCC como medio. Por ejemplo, los datos encontrados en la optimización del cloruro de tionilo muestran la tendencia de cada uno de los átomos a separarse de la molécula en cuanto la energía del sistema se estabiliza, por lo que habría un incremento en la posibilidad de interacción que pueda tener esta especie con otras moléculas (ver figura 7).

Hasta este punto se evidencian resultados bastante interesantes que permiten tener claridad con respecto al trabajo en general; sin embargo, se hace uso de una nueva herramienta para confirmar los datos obtenidos por medio del análisis poblacional y el análisis de orbitales de frontera. Para esto se implementa el paquete computacional WinMopac, empleando método semiempírico y tipo de cálculo AM1 con el objetivo de obtener datos termodinámicos para cada una de las moléculas. Los resultados son utilizados en la construcción de una gráfica de energía que permita seguir el curso de reacción de los sistemas, correlacionando la energía de formación de reactivos, intermediarios y productos, además de la influencia que pueda tener el medio empleado en la misma.

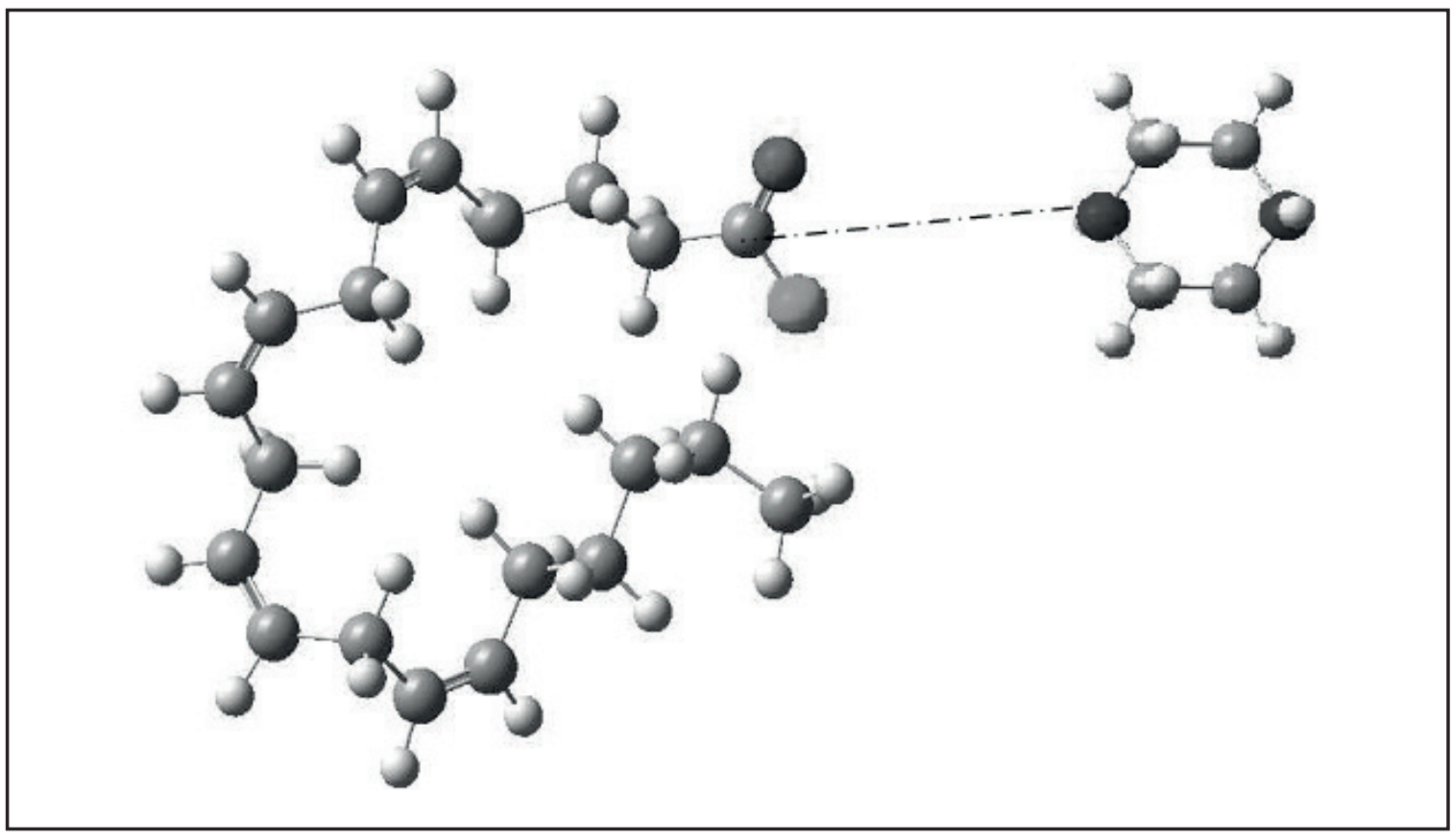

Figura 5. Reacción II para derivados de ácido araquidónico. Modelada con Gaussian View.

Fuente: elaboración propia. 
Finalmente, se procede con el análisis del comportamiento propio del sistema y su comparación con las demás reacciones para confirmar o rechazar el camino propuesto.

Es conocido que este tipo de métodos (semiempíricos) no puede arrojar unos valores confiables en lo que corresponde a valores de optimización, análisis poblacional y Eigenvalues; pero la teoría asegura que en lo que tiene que ver con variables termodinámicas, en especial la energía de activación, el margen de error es bajo y el ahorro de tiempo dispuesto para el cálculo se reduce considerablemente. En los valores utilizados para la construcción de las gráficas (ver figuras 7 y 8) se tuvo en cuenta la influencia del reactivo que cumple el papel de activante de la molécula inicial, para conocer el papel que este pueda llegar a desempeñar en el desarrollo de la reacción.

En la gráfica 8 se observa que algunas de las reacciones requieren menor energía, así que ellas serán las que tengan mayores posibilidades de suceder (ver gráfica 9). El procedimiento se aplicó para la totalidad de las reacciones diseñadas y se observó congruencia con respecto a los datos obtenidos por medio del análisis poblacional y de orbitales de frontera. Teniendo en cuenta los mínimos de energía se propone privilegiar las reacciones con valores finales bajos y que además muestren caída energética en la parte final de la gráfica o, dicho de

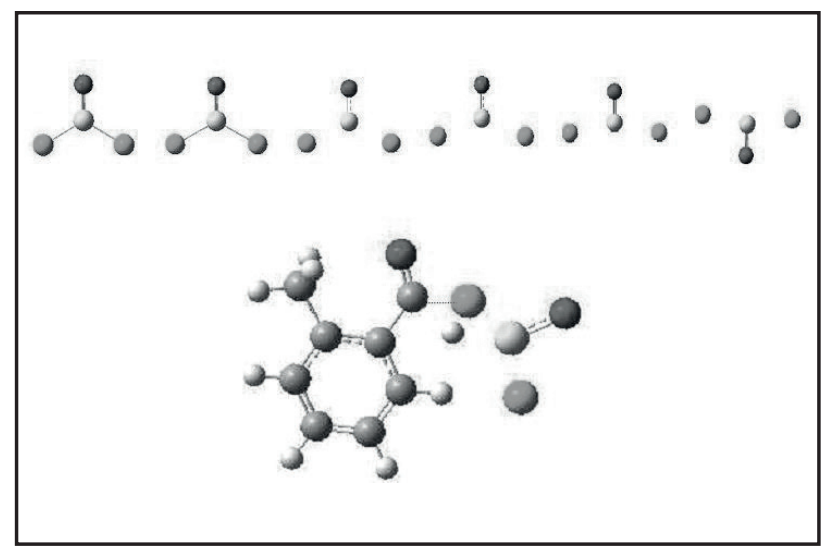

Figura 7. Arriba, optimización de cloruro de tionilo. Abajo, reacción 15 paraariletanolaminas. Modelación realizada con Gaussian View.

Fuente: elaboración propia.
1)

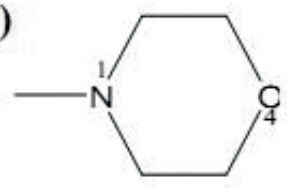

\begin{tabular}{|c|c|c|c|c|}
\hline \multicolumn{3}{|c|}{ Reactivo I } & HOMO & LUMO \\
24 & 25 \\
\cline { 1 - 3 }$N^{\circ}$ & Atomo & Orbital & -0.35237 & 0.25039 \\
\hline 1 & $\mathrm{~N}$ & $2 \mathrm{PZ}$ & 0.38294 & -0.00293 \\
\hline 4 & $\mathrm{O}$ & $2 \mathrm{PZ}$ & $\mathbf{1} 08067$ & 0.00462 \\
\hline
\end{tabular}

\begin{tabular}{|c|c|c|c|c|}
\hline \multicolumn{3}{|c|}{$\begin{array}{c}\mathrm{AA} \text { activado } \mathrm{SOCl}_{2} \\
\mathrm{HF}\end{array}$} & $\begin{array}{l}\text { HOMO } \\
24\end{array}$ & $\begin{array}{c}\text { LUMO } \\
25\end{array}$ \\
\hline$N^{\circ}$ & Átomo & Orbital & -0.43294 & 0.12677 \\
\hline 4 & C & $2 P Z$ & 0.00000 & 0.40597 \\
\hline 10 & $\mathrm{Cl}$ & $2 \mathrm{PZ}$ & 0.00000 & 0.04834 \\
\hline
\end{tabular}

\begin{tabular}{|c|c|c|c|c||}
\hline \multicolumn{3}{|c|}{$\begin{array}{c}\text { AA activado DCC } \\
\text { HF }\end{array}$} & $\begin{array}{c}\text { HOMO } \\
84\end{array}$ & $\begin{array}{c}\text { LUMO } \\
85 \\
0.1891\end{array}$ \\
\hline$N^{\circ}$ & Átomo & Orbital & -0.43945 & 0.189 \\
\hline 4 & $\mathrm{C}$ & $2 \mathrm{PZ}$ & 0.00000 & 0.41320 \\
\hline 10 & $\mathrm{O}$ & $2 \mathrm{PZ}$ & 0.00002 & -0.15566 \\
\hline
\end{tabular}

\begin{tabular}{|c|c|c|c|c|}
\hline \multicolumn{3}{|c|}{$\begin{array}{c}\text { AA activado } \mathrm{CH}_{3} \mathrm{CN} \\
\mathrm{HF}\end{array}$} & $\begin{array}{l}\text { HOMO } \\
84\end{array}$ & $\begin{array}{l}\text { LUMO } \\
85\end{array}$ \\
\hline$N^{0}$ & Átomo & Orbital & -0.41204 & 0.16358 \\
\hline 4 & C & $2 \mathrm{PZ}$ & 0.00010 & 0.36903 \\
\hline 1 & C & $2 P Z$ & $=0.00012$ & -0.00634 \\
\hline
\end{tabular}
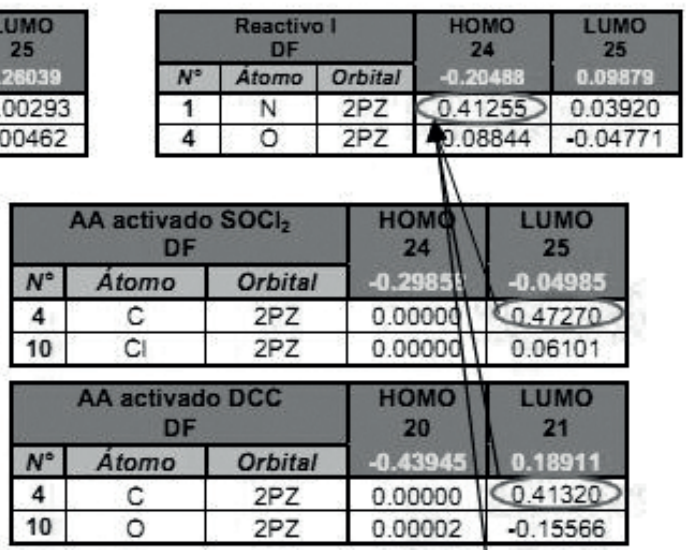

\begin{tabular}{|c|c|c|c|c|}
\hline \multicolumn{3}{|c|}{$\begin{array}{c}\text { AA activado } \mathrm{CH}_{3} \mathrm{CN} \\
\text { DF }\end{array}$} & $\begin{array}{c}\text { HOMO } \\
20\end{array}$ & $\begin{array}{l}\text { LUMO } \\
21\end{array}$ \\
\hline$N^{0}$ & Átomo & Orbital & -0.24743 & -0.01873 \\
\hline 4 & C & $2 P Z$ & 0.00001 & 0.43858 \\
\hline 1 & C & $2 P Z$ & -0.00004 & -0.02833 \\
\hline
\end{tabular}

Figura 6. Tablas de correlación de análisis poblacional para reacción II de derivados de ácido araquidónico 


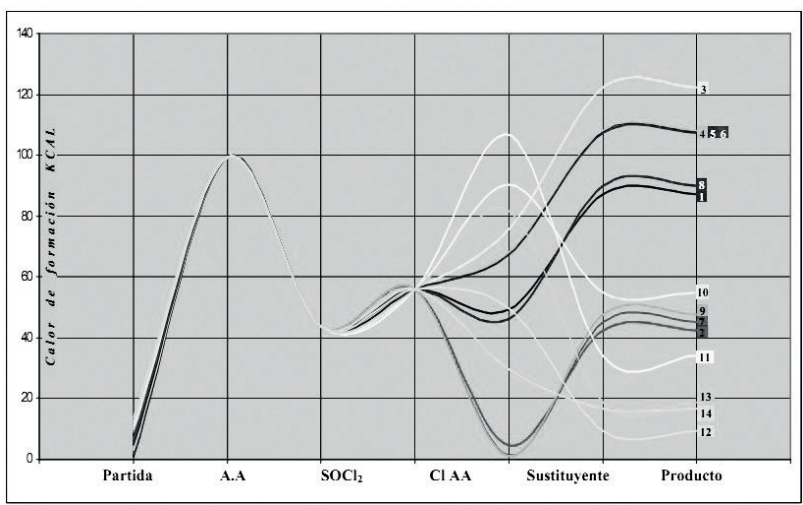

Figura 8. Gráficas del curso de reacción para derivados de ácido araquidónico.

Fuente: elaboración propia.

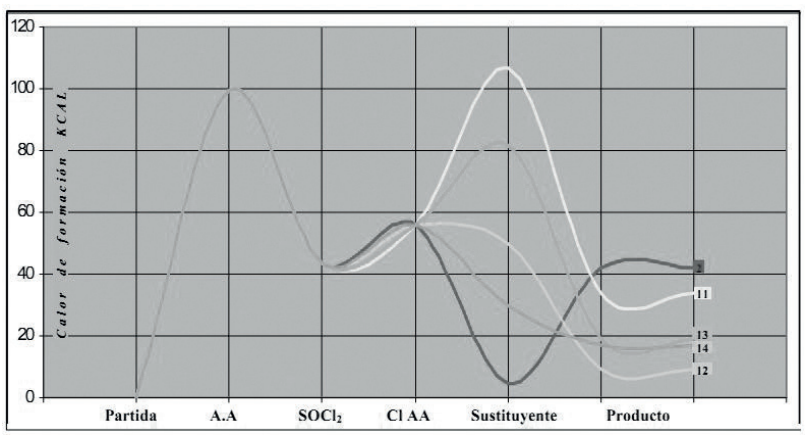

Figura 9. Gráficas para las reacciones de mínima energía. Curso de reacción para derivados de ácido araquidónico.

Fuente: elaboración propia.

otra manera, las reacciones en las que la energía de los productos es menor que la de los sustituyentes. Quienes cumplen con estos parámetros, en el caso de los derivados de ácido araquidónico, serían las reacciones 2, 11, 12, 13 y 14 . En cuanto a las reacciones con ariletanolaminas, se muestra disparidad en cuanto a las reacciones 8 y 9 puesto que en el análisis poblacional y de orbitales se dice que las magnitudes arrojadas no muestran éxito en la ocurrencia de reacción, pero, en la parte de las gráficas, estas quedan situadas dentro de la región de más baja energía. Se recomienda en este caso elaborar las gráficas de barreras de activación para que con ello se logre comprender este caso y privilegiar las reacciones 5, 6 y 7 en el momento de la síntesis.

\section{Conclusiones}

- A partir de la implementación de técnicas computacionales, es posible estudiar un sistema en particular y actuar de forma adecuada con el mismo, en la predicción de reacciones propuestas o ajustando el proceso que se viene dando para lograr mejores resultados.

- Se comprueba que la mayor parte de reacciones propuestas para análogos de ácido araquidónico y ariletanolamidas cuentan con altas probabilidades de tener éxito en la síntesis experimental. Se recomienda dar prioridad a las reacciones $2,7,11,12$, 13 y 14 de derivados de la anandamida y 1, 2, 3, $4,6,7,11,12,13,14,15$ y 16 de ariletanolamidas, por la magnitud y concordancia entre los cálculos efectuados.

-Los resultados muestran que se hace absolutamente necesario tener las moléculas de partida activadas, para conseguir un curso de reacción mejor; al parecer el medio que influye de mejor manera en esta parte de los procedimientos sería el de NN-diciclohexilcarboiimida (DCC), pues la cantidad de energía que se requiere para cada una de las reacciones es menor que la que se necesita con otros medios.

-Las reacciones que no registraron valores altos tienen algunas posibilidades de generar los compuestos esperados, pero puede ser que consigan mejores resultados si se someten a condiciones diferentes en cuanto a presión, temperatura y cantidad de reactivo.

-Las gráficas de correlación energética solamente sirven para comprender parcialmente cada una de las reacciones. Se recomienda que para efectos de conocer de mejor manera el comportamiento de cada una de ellas se construya un perfil energético, en el que se muestren las barreras de activación que necesita para superar cada camino propuesto.

- La base matemática con la que se trabajó fue la 3-21G, tanto en metodología Ab-Initio como en Funcionales de Densidad, debido a que era la base que presentaba mejor comportamiento con los recursos que se tenían; sin embargo, si se requiere 
hacer un cálculo más preciso se recomienda subir un nivel de base o adicionar funcionales difusas al cálculo.

-Es necesario que el proyecto curricular de Licenciatura en Química de la Universidad Distrital Francisco José de Caldas fortalezca el espacio académico de Química Teórica y Química Cuántica, elevando así el nivel disciplinar a estándares internacionales.

\section{Referencias}

Atmaram, D., Khanolkar, V., Vasiliki, A., Sonyuan, L., Adams, W., Hill, G....Makriyannis, A. (1996). Head group analogs of arachidonylethanolamide, the endogenous cannabinoid ligand. Journal of Medicinal Chemistry, 39(22).

Avendaño, A. C. \& Morales, L. (1995). Estudio cuántico mecánico de la reacción entre3-amino-aril2etoxicarboniltiofenosycetonasaromáticas $\alpha y \beta$ insaturadas. Proyecto Curricular de Licenciatura en Química, Universidad Distrital. Tesis de grado.

Cadogan, A., Kulkarni, R., Alexander, S., \& Kendall, D. (1997). Anandamide inhibits striatal dopamine release in the rat in vivo and in vitro. The Pharmacologist, 39, 90 (abstract).

Cotton, A. (1991). La teoría de grupos aplicada a la química. Ed. Limusa.

Cramer, J. (2002). Essentials of Computational Chemistry, theories and models. John Wiley \& Sons.

Devane, W. A., Hanus, L., Breuer, A., Pertwee, R. G., Stevenson, L. A., Griffin, G... Mechoulam, R. (1992). Isolation and structure of a brain constituent that binds to the cannabinoid receptor. Science 258, 1946-1949 (abstract).

Galeano, N., Bernal, A. \& Arias, R. (1999). Estudio mecano-cuántico para la obtención de oxa
[1,1,1] propelano. Proyecto Curricular de Licenciatura en Química, Universidad Distrital. Tesis de grado.

Gómez, A., Sarmiento, D. \& Yosa, J. (2001). Construcción teórica de espectros infrarrojos para clorometano, agua y metano, basados en la simetría molecular y los métodos de aproximación semiempíricos. Proyecto Curricular de Licenciatura en Química, Universidad Distrital. Tesis de grado.

Gómez, D. A. (2007). Exploración teórica de la interacción entre etanol y ácido sulfúrico a diferentes temperaturas. Proyecto Curricular de Licenciatura en Química, Universidad Distrital. Tesis de grado.

Khanolkar, A. \& Makrillanis, A. (1999). Structure-activity relationships of anandamida, an endogenous canabinoid ligand. Life Sciences, 65(6/7), 107-616.

Levine, I. N. (2001). Química cuántica. 5a ed. Prentice Hall.

Manual de usuario. (2002). Hyperchem release, tools for molecular modeling. Hypercube.

Matsuda, L., Bonner, T. \& Lolait, S. (1990). Structure of a cannabinoid receptor and functional expression of the cloned DNA. Nature, 346, 561-564.

Mechoulam, R. \& Fride, E. (1995). The unpaved road to the endogenous brain cannabinoid ligands, the anandamides in "Cannabinoid Receptors" (pp. 233-258) (ed. R. Pertwee). London: Academic Press.

Mechoulam, R., Fride, E., \& Hanu, L. (1997). Anandamida may mediate sleep induction. Nature, 389, 25-26.

Montero, L. A. (1991). Molecular orbitals by semiempirics SCF-MO, methods. Version 1.00, April, 1991* (6), copyright Universidad de La Habana. 
Piomelli, D. (2003). The molecular logic of endocannabinoid signaling, Nature, Neuroscience, $4,873-884$.

Randall, M. D., Alexander, S., et al. (1999). An endogenous cannabinoidis and endotheliumderived vasorelaxant. Biochem Biofys Res Commun, 229, 114-120.

Pinzón, S. (1999). Exploración experimental y estudio mecano-cuántico de una ruta de síntesis para la obtención de 1-fenil-imino[1,1,1] propelano. Proyecto Curricular de Licenciatura en Química, Universidad Distrital. Tesis de grado.

Quijano, J. \& Vélez, O. E. (2004). Introducción a la Química Computacional. Universidad Nacional de Colombia, sede Medellín.
Quiroga, J. (1999). Estudio mecano-cuántico para la obtención de tio $[1,1,1]$ propelano. Proyecto Curricular de Licenciatura en Química, Universidad Distrital. Tesis de grado.

Rincón, F. N. (2007). Software especializado y su empleo en la enseñanza de la química orgánica: dos casos puntuales. Proyecto Curricular de Licenciatura en Química, Universidad Distrital. Tesis de grado.

Vogel, Z., Barg, J., Levy, R., Saya, D., Heldman, E., \& Mechoulam, R. (1993). Anandamida, a brain endogenous compound, interacts specifically with cannabinoid receptors and inhibits adenylate cyclase. J. Neurochem, 61, 352-355.

Woodward, R. B. \& Hoffmann, R. (1972). La conservación de la simetría orbital. Madrid: Alhambra. 